Srmmons, D. A. R. (1957). J. gen. Microbiol. 17, 650-657.

\title{
The Hexose Constituents of some Shigella Polysaccharide Hydrolysates
}

\author{
By D. A. R. SIMMONS \\ Department of Bacteriology of the University of St Andrews, \\ Queen's College, Dundee
}

\begin{abstract}
SUMMARY: Polysaccharides obtained from twelve previously unextracted Shigella strains were hydrolysed and analysed chromatographically to determine their hexose constituents. The polysaccharides extracted from eight strains of the Flexner group (NCTC strains 3, 4833, 8205, 8206, 8201, 8207, 2245 and 4839), all contained the same hexose constituents, namely glucosamine, glucose and rhamnose. Shigella shigae (NCTC 8005) polysaccharide contained glucosamine, galactose and rhamnose. S. sonnei (NCTC 8220) polysaccharide contained glucosamine, galactose and glucose. S. schmitzi (NCTC 8218) polysaccharide contained glucosamine, galactose, glucose and rhamnose. S. alkalescens (NCTC 7925) polysaccharide contained glucosamine, galactosamine, glucose and galactose.
\end{abstract}

The introduction of carbohydrate paper chromatography by Partridge (1948a) provided a simple method of confirming the chemical studies of Shigella polysaccharides by Morgan (1931, 1932, 1936, 1937), Morgan \& Partridge (1940, 1941) and Goebel, Binkley \& Perlman (1945), and of extending these studies to other Shigella polysaccharides. Partridge $(1948 b)$ confirmed the presence of glucosamine, galactose and rhamnose in the hydrolysis products of Shigella shigae polysaccharide extracted by the diethyleneglycol method. Jesaitis \& Goebel (1952) identified glucosamine, galactose and glucose in the hydrolysis products of $S$. sonnei polysaccharide extracted by the infusion of phenolkilled organisms with warm distilled water. Slein \& Schnell (1952), and Slein (1952), identified glucosamine, glucose and rhamnose in the hydrolysis products of $S$. flexneri type 3 polysaccharide. Davies (1955) identified glucosamine, galactose, glucose and rhamnose in the hydrolysis products of $S$. schmitzi polysaccharide and galactosamine, glucosamine, galactose and glucose in $S$. alkalescens polysaccharide. The trichloroacetic acid method of extraction was used in the preparation of the last two polysaccharides. Aldoheptoses have been identified in a lipopolysaccharide extracted from $S$. sonnei by Jesaitis \& Goebel (1952), from a phosphorus-containing polysaccharide extracted from $S$. flexneri type 3 by Slein \& Schnell (1953), and in a lipopolysaccharide extracted from $S$. shigae by Davies, Morgan \& Record (1955).

In the present work the trichloroacetic acid method of extraction (Boivin \& Mesrobeanu, 1934, 1937) was used because it is now generally accepted that antigenic preparations can be obtained in this way from the majority of Gramnegative bacilli (Boivin \& Mesrobeanu, 1984, 1937; MacKenzie, Pike \& Swinney, 1940; Davies, 1955), although it fails to remove the analogous substances from Pasteurella pestis (Girard, 1941), and some Escherichia coli strains (MacPherson, 1956). When applicable the diethyleneglycol method 


\section{Shigella polysaccharides}

(Morgan, 1937) is the most satisfactory method as this solvent is effective at neutrality and at normal temperatures. Unfortunately it is not generally applicable to the Shigella group as the yield with some strains is poor and with Shigella flexneri type 6 no antigen can be recovered at all (Goebel, Binkley \& Perlman, 1945). Trichloroacetic acid extracts are not, however, homogeneous but consist mainly of two components, the one being a polypeptide-phospholipid-polysaccharide complex or antigenic complex, and the other free serologically-active polysaccharide (Davies, Morgan \& Mosimann, 1954). The yields of polypeptide-phospholipid-polysaccharide complex and of polysaccharide are not so high as with other methods of extraction but the preparations are less heavily contaminated with other cell constituents, e.g. phenol extracts contain a certain amount of simple protein (Morgan \& Partridge, 1941) and pyridine extracts comparatively large amounts of nucleic acids (Goebel et al. 1945).

The object of the present work was to identify by chromatographic methods the hexose constituents of the polysaccharides from: (1) a representative group of Flexner strains, one strain being selected from each of the eight main types; and (2) four other members of the genus Shigella.

\section{METHODS}

The organisms used in this investigation were the following, the numbers in brackets being the NCTC numbers: Shigella flexneri type 1 a (3); S. flexneri type 2a (4833); $S$. flexneri type $3(8205) ; S$. flexneri type 4a (8206); $S$. flexneri type 5 (8201); $S$. flexneri type 6 (8207); Flexner X (Andrewes \& Inman; 2245); Flexner Y (Andrewes \& Inman; 4839); S. shigae (8005); S. sonnei (8220); S. schmitzi (8218); S. alkalescens (7925).

Preparation of organisms. The purity of each culture used for mass inoculation was checked by morphological, biochemical and serological tests and its smoothness confirmed by colonial appearance and salt stability. The cultures were grown on ox-heart extract agar at $37^{\circ}$ for $20 \mathrm{hr}$. and washed off in $\mathbf{0 . 9} \%$ $(\mathrm{w} / \mathrm{v})$ saline. The organisms were washed a further three times in $\mathbf{0 . 9} \%(\mathrm{w} / \mathrm{v})$ saline and then twice in distilled water. The bacterial sludge obtained after the last wash was suspended in $30 \mathrm{ml}$. distilled water and the organisms killed by adding $10 \mathrm{vol}$. acetone at $-15^{\circ}$. After two more washes in acetone the organisms were dried in vacuo over conc. $\mathrm{H}_{2} \mathrm{SO}_{4}$ and stored at $0^{\circ}$. Each Flexner mass culture, harvested from five hundred 4-in. Petri plates, yielded approximately $6.0 \mathrm{~g}$. acetone-dried bacteria, while each mass culture of the other members of the genus, harvested from two hundred and fifty 4-in. Petri plates, yielded approximately $3.0 \mathrm{~g}$. The bacteriological purity of each plate was checked by inspection and by slide agglutination with the appropriate standard antiserum obtained from the Standards Laboratory, Colindale.

Trichloroacetic acid extraction of bacteria. The acetone-dried bacteria were extracted for $4 \mathrm{hr}$. by the method of Boivin \& Mesrobeanu (1934) with N-trichloroacetic acid. After separation of the bacteria by centrifugation the extracts were dialysed to remove trichloroacetic acid and fractionated with ethanol to recover the polypeptide-phospholipid-polysaccharide complex and 
the polysaccharide. These compounds precipitated from aqueous solution on the addition of 2 vol. ethanol and were stored till use in $75 \%(v / v)$ ethanol in water at $0^{\circ}$.

The preparation of polysaccharides. The polysaccharides were prepared from the mixture of polypeptide-phospholipid-polysaccharide complex and polysaccharide $(1 \%, w / v$, aqueous solution) by partial degradation with $1 \%(v / v)$ acetic acid at $100^{\circ}$. After $4 \mathrm{hr}$. hydrolysis the acid-insoluble precipitate which formed was separated by centrifugation and the supernatant fluid cooled to $0^{\circ}$ and extracted with ether. The material soluble in the aqueous phase proved to be polysaccharide. It was recovered from solution by the addition of $2 \mathrm{vol}$. ethanol and was repeatedly precipitated from aqueous solution by ethanol.

The degradation of the polysaccharide component of trichloroacetic acid extracts. Each polysaccharide solution was refluxed for $6 \mathrm{hr}$. in $0.25 \mathrm{~N}-\mathrm{HCl}$ and then de-ionized in a mercury cathode de-salter until free from chloride and sulphate. This ion-free solution of hexoses was concentrated and analysed by chromatographic methods.

Chromatography. The descending method was employed. Whatman No. 4 filter paper was used in all experiments.

Solvents. The following three solvents were used in the analysis of each polysaccharide: $n$-butanol + acetic acid + water $(4: 1: 5, \mathrm{v} / \mathrm{v}$; Slotta \& Primosigh, 1951); $n$-butanol + ethanol + water (10:1:2, v/v; Williams \& Bevenue, 1951); ethyl acetate + acetic acid + water (3:1:3, v/v; Jermyn \& Isherwood, 1949).

Identifying reagents. In recent years a number of rare or unidentified sugars have been isolated from bacterial polysaccharides (Lüderitz \& Westphal, 1952; Dudman \& Wilkinson, 1956; Davies, 1956; Strange \& Dark, 1956). In order to be able to detect all classes of carbohydrate and to decrease the possibility of some sugars remaining unidentified, a wide range of identifying reagents was used. The identity of an unknown sugar was considered to be established when its $R_{\theta}$ value corresponded with that of a known control in several different solvents and when its colour reactions with eight identifying reagents also corresponded with those of the same control. The following reagents were used in the chromatographic analysis of each polysaccharide: benzidine reagent (Horrocks, 1949); aniline hydrogen oxalate reagent (Horrocks \& Manning, 1949); ammoniacal silver nitrate reagent (Partridge, 1948a); naphthoresorcinol reagent (Partridge, 1948a) modified as follows: 1 vol. $0.5 \%(\mathrm{w} / \mathrm{v})$ ethanolic naphthoresorcinol added to 1 vol. $0 \cdot 1 \mathrm{~N}-\mathrm{HCl}$. By decreasing the amount of acid it was possible to prevent the charring of chromatograms which tends to occur when higher concentrations are used. The colour differentiation obtained with different sugar types was unimpaired); ninhydrin reagent (Partridge, 1948a); 3:5-dinitrosalicylic acid reagent (Williams \& Bevenue, 1951); $\beta$-naphthylamine reagent (Novellie, 1950); iodine vapour reagent (Greenway, Kent \& Whitehouse, 1953).

Special techniques. The separation of glucosamine and galactosamine by paper chromatography is not always easy. Two methods were used to differentiate these hexosamines. The first depends on the utilization of glucosamine as substrate by hexokinase in the presence of adenosine-5-triphosphate. 
The method was described by Slein (1952). The second method, which is more efficient, depends on the conversion of 2-amino sugars to their corresponding parent pentoses by ninhydrin in pyridine buffer. The technique used was as described by Stoffyn \& Jeanloz (1954) except that the sealed ampoules were heated at $100^{\circ}$ for $1 \mathrm{hr}$. About $50 \mu \mathrm{g}$. of each hexosamine treated with ninhydrin reagent in pyridine buffer were applied to the chromatograms.

Two-dimensional chromatography and elution techniques were used to confirm the results obtained with the above methods.

\section{RESULTS}

The treatment of acetone-dried organisms of twelve Shigella strains with trichloroacetic acid resulted in each instance in the recovery of polypeptidephospholipid-polysaccharide complex and polysaccharide. The amount of material so obtained varied from organism to organism, being as high as 6.7\% of the dry weight of bacteria with Shigella flexneri type 1 a, and as low as $4.4 \%$ of the dry weight with $S$. flexneri type 6 .

\section{The chemical nature of the trichloroacetic acid extracts}

On partial hydrolytic degradation with acetic acid each trichloroacetic acid extract was shown to consist of three components. In their gross chemical and physical properties these components were analogous to those already studied by Morgan \& Partridge (1940) and by Goebel et al. (1945). Component I was insoluble in acetic acid and ether and on prolonged hydrolysis with $\mathrm{N}-\mathrm{HCl}$ yielded a number of amino acids but no monosaccharides; this component was considered to be a polypeptide. Component II was ethersoluble and contained phosphorus when examined by the method of Hanes \& Isherwood (1949); it was considered to be a phospholipid. Component III was soluble in acetic acid and insoluble in ether. On hydrolysis with $\mathrm{N}-\mathrm{HCl}$ it yielded a number of monosaccharides and no amino acids; it was considered to be a polysaccharide.

\section{Chromatographic analysis}

Each of the twelve antigenic complexes under investigation and their polypeptide, phospholipid and polysaccharide components was examined chromatographically, using the solvents and identifying reagents described above to demonstrate that they were not contaminated with free amino acids or sugars. The hydrolysed polypeptides were examined chromatographically and shown to be free from sugars, indicating that the polypeptides were free from polysaccharide contamination. Similarly, the hydrolysed polysaccharides were examined chromatographically and shown to be free from amino acids, indicating that the polysaccharides were free from polypeptide contamination. About $500 \mu \mathrm{g}$. of each component were applied to the chromatogram.

The hexose constituents of the Shigella polysaccharides

Shigella flexneri type 1 a polysaccharide hydrolysate contained three monosaccharide constituents. Each of these constituents reacted with benzidine, aniline hydrogen oxalate, 3:5-dinitrosalicylic acid, ammoniacal silver nitrate 
and $\beta$-naphthylamine, giving the typical colour reactions of an aldose. The first constituent also gave a positive ninhydrin reaction and is therefore an amino sugar. It corresponded in $\boldsymbol{R}_{\boldsymbol{\theta}}$ with a control of glucosamine hydrochloride in a number of different solvents and was utilized as substrate in the hexokinase reaction, thus confirming its identity. The second constituent did not react with the ninhydrin, naphthoresorcinol or iodine reagents but corresponded in $\boldsymbol{R}_{\theta}$ with a control of glucose in several different solvents; it also reacted as substrate in the hexokinase reaction, thus confirming its identity. The third constituent gave a positive naphthoresorcinol reaction and its colour reactions with benzidine and aniline hydrogen oxalate also suggested that it was a substituted pentose. It corresponded in $\boldsymbol{R}_{\boldsymbol{\sigma}}$ value with a control of rhamnose in several solvents. The results of a similar series of experiments with each of the other seven Flexner polysaccharide hydrolysates revealed that they likewise consisted of the same three compounds, namely glucosamine hydrochloride, glucose and rhamnose. The identity of the 2-amino sugar in the hydrolysate of $S$. flexneri type 2 a polysaccharide was confirmed by demonstrating its utilization as substrate in the hexokinase reaction. The 2-amino sugars in the hydrolysates of the polysaccharides from $S$. flexneri types 3, 4a, 5 and 6 and from Flexner $X$ and Flexner $Y$ were converted to arabinose by the ninhydrin reaction in pyridine buffer. The 2-amino sugar present in these Flexner polysaccharides therefore seems most likely to be glucosamine hydrochloride.

By the same methods the hexoses present in the other Shigella polysaccharide hydrolysates were determined as follows: Shigella shigae polysaccharide hydrolysate contained glucosamine hydrochloride, galactose and rhamnose; $S$. sonnei polysaccharide hydrolysate contained glucosamine hydrochloride, galactose and glucose; $S$. schmitzi polysaccharide hydrolysate contained glucosamine hydrochloride, galactose, glucose and rhamnose; $S$. alkalescens polysaccharide hydrolysate contained glucosamine hydrochloride, galactosamine hydrochloride, glucose and galactose. The identity of the 2-amino sugars was confirmed by converting them to pentoses with ninhydrin reagent in pyridine buffer.

Table 1. The constituent hexoses of the Shigella polysaccharides

$\quad \begin{aligned} & \text { Shigella } \text { strain from which } \\ & \text { polysaccharide was extracted }\end{aligned}$
Shigella flexneri type 1 a
S. flexneri type 2a
S. flexneri type 3
S. flexneri type 4a
S. flexneri type 5
S. flexneri type 6
Flexner X (Andrewes \& Inman)
Flexner Y (Andrewes \& Inman)
S. shigae
S. sonnei
S. schmitzi
S. alkalescens

$\begin{array}{cc}\text { Glucos- } & \\ \text { amine } & \text { Galactose } \\ + & - \\ + & - \\ + & - \\ + & - \\ + & - \\ + & - \\ + & - \\ + & - \\ + & + \\ + & + \\ + & + \\ + & +\end{array}$

Glucose
+
+
+
+
+
+
+
+
+
+
+
+

$\begin{array}{cc}\text { Rhamnose } & \text { Galactos } \\ \text { amine } \\ + & - \\ + & - \\ + & - \\ + & - \\ + & - \\ + & - \\ + & - \\ + & - \\ + & - \\ - & - \\ + & - \\ - & +\end{array}$


Arabinose was formed from the 2-amino sugar present in each polysaccharide hydrolysate so that this hexosamine seems most likely to be glucosamine hydrochloride. In the case of the Shigella alkalescens polysaccharide hydrolysate, lyxose was also formed from the second 2-amino sugar so this hexosamine seems to be galactosamine. Control specimens of glucosamine and galactosamine hydrochlorides were treated with ninhydrin reagent in pyridine buffer as described above under identical experimental conditions to demonstrate their conversion to arabinose and lyxose respectively. Table 1 shows the constituent monosaccharides of the Shigella polysaccharides which have been studied in this investigation.

\section{CONCLUSIONS}

The eight Flexner polysaccharides investigated all possess identical monosaccharide constituents. The other Shigella polysaccharides differ in their monosaccharide constituents not only from the Flexner group as a whole but also from one another. In other words, the polysaccharides of the serologically heterogeneous Flexner group contain identical monosaccharides while the polysaccharides of the serologically homogeneous members of the genus Shigella contain different monosaccharides. The finding that $S$. alkalescens (NCTC 7925) polysaccharide contains galactosamine confirms the observation of Davies (1955). The absence of this hexosamine from the polysaccharides of the other Shigella types may be of taxonomic significance. It is of interest to note that the Shigella Commission Report (1951) removes this organism from the genus Shigella and places it in the genus Escherichia on the basis of serological evidence.

The chief value of the present work is that the number of Flexner polysaccharides examined has now been extended to include at least one from each Flexner type. In the case of the polysaccharides extracted from the other members of the genus Shigella the findings of previous workers have been confirmed using, in all but two instances, a different method of extraction. Goebel et al. (1945) reported the presence of a hexosamine (estimated as glucosamine) in the polysaccharides from $S$. flexneri types $1 \mathrm{a}, 2 \mathrm{a}, 3$ and 6 . Slein \& Schnell (1952) and Slein (1952) identified glucosamine, glucose and rhamnose in the polysaccharide from a strain of $S$. flexneri type 3. These findings are confirmed here chromatographically by using a different method of polysaccharide extraction; the hexose constituents of the polysaccharides from strains of $S$. flexneri types $4 \mathrm{a}$ and 5 and Flexner $\mathrm{X}$ and $\mathrm{Y}$ are identified for the first time. The findings of Morgan (1931, 1932, 1936, 1937), Morgan \& Partridge (1940, 1941) and Partridge $(1948 b)$ in respect of the hexose constituents of $S$. shigae polysaccharide are confirmed with a different strain of $S$. shigae. The polysaccharide from the strain of $S$. sonnei examined contains the same hexose constituents as the strain studied by Jesaitis \& Goebel (1952) who used a different method of extraction. The polysaccharides from the strains of $S$. alkalescens and $S$. schmitzi examined contained the same hexose constituents as those examined by Davies (1955), using the same method of extraction. 
This paper forms part of an M.D. thesis presented by D. A. R. Simmons to the University of St Andrews, St Andrews. I wish to express my thanks to Professor W. J. Tulloch and Dr T. Sommerville for their interest and encouragement throughout this work; and to Professor A. D. Walsh and Dr R. Roger of the Chemistry Department, Queen's College, Dundee, for accommodation granted to me for the completion of this work.

\section{REFERENCES}

Borvin, A. \& Mesnobeanu, L. (1934). Remarques concernant la technique d'extraction du complexe polysaccharidique antigénique renfermé dans le bacille d'Aertrycke. C.R. Soc. Biol., Paris, 115, 304.

Boivin, A. \& Mesrobeanu, L. (1937). Recherches sur les toxines des bacilles dysentériques. Sur la nature et sur les propriétés biologiques des principes toxiques susceptibles de se recontrer dans les filtrats des cultures sur bouillon bacille de Shiga. C.R. Soc. Biol., Paris, 124, 442.

Davies, D. A. L. (1955). The specific polysaccharides of some Gram-negative bacteria. Biochem. J. 59, 696.

Davies, D. A. L. (1956). A specific polysaccharide of Pasteurella pestis. Biochem. J. 63, 105.

Davies, D. A. L., Morgan, W. T. J. \& Mosimann, W. (1954). Studies in immunochemistry. 13. Preparation and properties of the ' $O$ ' somatic antigen of Shigella dysenteriae (Shiga). Biochem. J. 56, 572.

Davies, D. A. L., Morgan, W. T. J. \& Record, R. B. (1955). Studies in immunochemistry. 15. The specific polysaccharide of the dominant ' $O$ ' somatic antigen of Shigella dysenteriae. Biochem. J. 60, 290.

Dudman, W. F. \& Wilkinson, J. F. (1956). The composition of the extracellular polysaccharide of Aerobacter-Klebsiella strains. Biochem. J. 62, 289.

Girard, G. (1941). Absence d'antigène glucido-lipidique chez le bacille de la peste et le bacille de la pseudo-tuberculose des rongeurs. C.R. Soc. Biol., Paris, 135, 1577 .

Goebel, W. F., Binkley, F. \& Perlman, E. (1945). Studies on the Flexner group of dysentery bacilli. J. exp. Med. 81, 315.

Greenway, R. M., Kent, P. W. \& Whitehouse, M. W. (1953). Detection of nonreducing carbohydrates in paper electrophoresis and chromatography. Research, Lond. 6, Suppl. no. 1.

Hanes, C. S. \& Isherwood, F. A. (1949). Separation of the phosphoric esters on the filter paper chromatogram. Nature, Lond. 164, 1107.

Horrocks, R. H. (1949). Paper partition chromatography of reducing sugars with benzidine as a spraying reagent. Nature, Lond. 164, 444 .

Horrocks, R. H. \& Manning, G. B. (1949). Partition chromatography on paper. Identification of reducing substances in urine. Lancet, $i, 1042$.

JERMYN, M. A. \& IsHerwOod, F. A. (1949). Improved separation of sugars on the paper partition chromatogram. Biochem. J. 44, 402.

JeSaITIS, M. A. \& Goeber, W. F. (1952). The chemical and antiviral properties of the somatic antigen of phase II Shigella sonnei. J. exp. Med. 96, 409.

LÜDERITz, O.\& WestPhaL, O. (1952). Utber bakterielle Reizstoffe: II Mitt.: Qualitative und quantitative papierchromatographische Bestimmung der Zuckerbausteine eines hochgereinigten Polysaccharid-pyrogens aus Colibakterien. Z. Naturf. $7 b, 548$.

Mackenzie, G. M., Pike, R. M. \& Swinney, R. E. (1940). Virulence of Salmonella typhimurium. J. Bact. 40, 171.

MacPeerson, C. R. (1956). Some observations on the antigenic structure of trichloroacetic acid extracts in Escherichia and the Paracolon group. J. gen. Microbiol. 14, 520. 
Morgan, W. T. J. (1931). A specific precipitating polysaccharide from B. dysenteriae (Shiga). Brit. J. exp. Path. 12, 62.

Morgan, W. T. J. (1932). The estimation of small amounts of a specific bacterial polysaccharide by the induction of anaphylaxis. Brit. J. exp. Path. 13, 342.

Morgan, W. T. J. (1936). Studies in immunochemistry. The preparation and properties of a specific polysaccharide from B. dysenteriae (Shiga). Biochem. $J$. 30, 909.

Morgan, W. T. J. (1937). Studies in immunochemistry. The isolation and properties of a specific antigenic substance from B. dysenteriae (Shiga). Biochem. $J$. 31, 2003.

Morgan, W. T. J. \& Partridge, S. M. (1940). Studies in immunochemistry. 4. The fractionation and nature of antigenic material isolated from Bact. dysenteriae (Shiga). Biochem. J. 34, 169.

Morgan, W. T. J. \& Partridge, S. M. (1941). Studies in immunochemistry. The use of phenol and of alkali in the degradation of antigenic material isolated from Bact. dysenteriae (Shiga). Biochem. J. 35, 1140.

Novellie, L. (1950). An improved method of detecting sugars on paper chromatograms. Nature, Lond. 166, 745.

Partridge, S. M. (1948a). Filter-paper partition chromatography of sugars. 1. General description and application to the qualitative analysis of sugars in apple juice, egg white and foetal blood of sheep. Biochem. J. 42, 238.

Partridge, S. M. $(\mathbf{1 9 4 8 b})$. Filter-paper partition chromatography of sugars. 2. An examination of the blood group $\mathbf{A}$ specific substance from hog gastric mucin and the specific polysaccharide of Bacterium dysenteriae (Shiga). Biochem. J. 42, 251.

Shigella Commission Report (1951). See International Bulletin of Bacteriological Nomenclature and Taxonomy, 1951, 1, 154.

SLEIN, M. W. (1952). A rapid method of distinguishing D-glucosamine from galactosamine in biological preparations. Proc. Soc. exp. Biol., N.Y. 80, 646.

Slein, M. W. \& Schnell, G. W. (1952). The specific polysaccharide of Shigella flexneri. Bact. Proc. p. 110.

Slein, M. W. \& Schnell, G. W. (1953). An aldoheptose phosphate in a polysaccharide isolated from Shigella flexneri. Proc. Soc. exp. Biol., N.Y. 82, 734.

Slotta, K. H. \& Primosigh, J. (1951). Amino-acid composition of crotoxin. Nature, Lond. 168, 696.

Stoffyn, P. J. \& JEanloz, R. W. (1954). Identification of amino-sugars by paper chromatography. Arch. Biochem. Biophys. 52, 373.

Strange, R. E. \& Dark, F. A. (1956). An unidentified amino-sugar present in cell walls and spores of various bacteria. Nature, Lond. 177, 186.

Williams, K. T. \& Bevenue, A. (1951). A simple technique for the identification of raffinose and sucrose by enzymatic hydrolysis on paper chromatograms and the subsequent separation of the hydrolysed products by paper chromatography. Science, 113, 582. 